hoped that this machine may prove to be of value in the teaching of symbolic logic, and that it will stimulate the interest of students in what otherwise tends to become a rather dull subject, and impress on them the mechanical nature of logical operations.

Acknowledgments are due to Ferranti, Ltd., for providing facilities for the construction of the machine and to Mr. R. Naylor for his work in assembling it. A fuller description of the machine and the underlying logical methods will be published elsewhere.

Department of Philosophy,

W. Mays

University of Manchester,

Manchester 13.

Ferranti, Ltd.,

Moston, Manchester 10. Sept. 16.

${ }^{1}$ Jevons, W. Stanley, Phil. Trans. Roy. Soc. 160, 497 (1870).

\section{Wetting and Contact-Angle Hysteresis}

THE study of contact angles and contact-angle hysteresis in the region of low contact angles is not easy owing to difficulties of technique. The contact angle, $\theta$, is given by

$$
\cos \theta=\frac{\gamma_{S A}-\gamma_{S L}}{\gamma_{L A}}
$$

where $\theta$ is the contact angle measured in the liquid and $\gamma_{S A}, \gamma_{S L}$ and $\gamma_{L A}$ are the surface free energies per unit area of the solid-air, solid-liquid and liquidair interfaces respectively.

In any practical case using a pure liquid, $\gamma_{S A}$ is not the true value, as the solid surface is modified by adsorption of the vapour of the liquid; and so in any contact-angle measurements it is the effective or modified value of $\gamma_{S A}$ which is of importance ${ }^{1-3}$. If a wetting agent is used, then all the interfacial energies may be modified by adsorption of the wetting agent. The interfacial energies considered in equation (1) therefore, will be the effective values. In general, equation (1) may be written :

$$
\gamma_{S A}-\gamma_{S L}=k \gamma_{L A},
$$

and wetting of a solid by a liquid is considered to occur when $k \geqslant 14,5$. The direct measurement of $k$ for such values is not possible as no finite contact angle exists.

Measurements of $k$ can, however, be made using an extension of the theory of the water repellency of porous surfaces ${ }^{8}$. If we consider a porous surface, then any liquid with a positive value of $k$ will penetrate to some degree into the surface. If we have a thin porous surface, then a drop of liquid with $k \geqslant 1$ will penetrate through the surface and, provided the porosity is sufficiently high and the drop sufficiently small, it will orient itself symmetrically on both sides of the surface, as is shown diagrammatically in the figure. In such a position the drop is completely stable and no spreading occurs along the solid portions of the porous surface even if they are continuous. That no spreading occurs along a fine filament in contact with a drop large in comparison with the filament diameter can readily be shown by suspending a drop of, say, liquid paraffin on a glass filament, when it will remain indefinitely if evaporation is eliminated. Spreading of a spherical drop over a cylindrical surface can only take place when the cylinder radius is greater than half the radius of

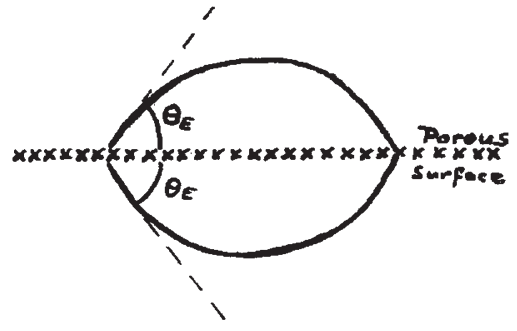

Liquid drop on thin porous surface

the drop, even though $k$ is equal to unity. The contact angle, $\theta_{E}$, of the diagram can be shown to be given by

$$
\cos \theta_{\boldsymbol{E}}=\frac{f^{2}}{2},
$$

where $f$ is the area of solid-air interface per unit geometrical area of the porous surface. Therefore, as $f$ and $\theta_{E}$ can be measured, $k$ can be determined.

In no case was a value of $k>1$ found and, using such liquids as benzene, aniline, diethyl phthalate, on fine nickel and copper structures ${ }^{7}$, the values of $\theta_{E}$ were found to be identical for all the organic liquids tested and were the same for expanding and contracting drops. Further, a determination of $f$ showed that $k$ was unity in all these cases. It was interesting to note that when a wetting agent was added to water, the maximum value of $k$ was again unity, and in this case also no contact-angle hysteresis was observable.

These results indicate that the liquid in such cases is, in fact, spreading over an adsorbed layer of itself on the solid, and that the energy changes on spreading are zero or negligible. That no hysteresis occurs indicates complete coverage of the solid surface by a layer of adsorbed molecules of the liquid. This is, of course, to be expected, since if the true value of $k$ is greater than 1 , then the liquid would have more affinity for the solid than for itself. In such a case complete coverage would take place.

A fuller account of this work, together with its application to the study of surface-active agents in wetting and detergency, will be presented in due course.

The Nickell Laboratories,

S. BAXTER

Monsanto Chemicals, Ltd., Ruabon, Wrexham, Denbighshire.

${ }^{1}$ Bangham and Razouk, Trans. Farad. Soc., B3, 1459 (1937).

${ }^{2}$ Doss and Rao, Ind. Acad. Sci., A, 7, 113 (1938).

${ }^{8}$ Yarnold and Mason, Proc. Phys. Soc., 62, (2), 121 (1949).

"Adam, "Physics and Chemistry of Surfaces", 209 (3rd edit., Oxford).

'Baxter and Cassie, J. Text. Inst., 36, T, 67 (1945).

- Cassie and Baxter, Trans. Farad. Soc., 40, 546 (1944).

7 Law, Rev. Sci. Instr., 19, 879 (1948).

\section{Induced Molecular Vibrational and Rotational Absorption}

IN view of recent advances in the technique of absorption measurements in the infra-red and micro-wave regions, I wish to direct attention to a method by which transitions normally forbidden in such absorption might be made to appear. The method is applicable, for example, to the vibration and rotation of homonuclear diatomic molecules and to the breathing mode of vibration of molecules like 